\title{
Duodenum/Ampulla/Jejunum/lleum Neuroendocrine Tumor pNX TNM Finding v7
}

National Cancer Institute

\section{Source}

National Cancer Institute. Duodenum/Ampulla/Jejunum/lleum Neuroendocrine Tumor

pNX TNM Finding v7. NCI Thesaurus. Code C90092.

Duodenum/ampulla/jejunum/ileum neuroendocrine tumor in which regional lymph nodes

cannot be assessed. (from AJCC 7th Ed.) 